\title{
Sistemi lineari completi su superficie di Enriques $\left({ }^{*}\right)$.
}

\author{
Matrizio Letizia (Trento)
}

Sunto. - Vedi Introduzione.

La presente ricerea è articolata in 4 sezioni. Nella prima vengono esposti in modo più preciso risultati che si trovano in ENRIQUES [1]. Tra l'altro viene provato che un sistema lineare completo senza parti fisse e con autointersezione positiva su una superficie di Enriques è irriducibile e o non ha punti base o ne ha esattamente due nel qual caso la curva generica del sistema è iperellittica e non singolare. Nella seconda sezione viene provato che se $|D| \grave{e}$ un sistema lineare completo senza parti fisse o punti base con $D^{2}>0$ su una superficie di Enriques $X$ il morfismo $\varphi_{D}: X \rightarrow \boldsymbol{P}^{h^{0}(D)-1}$ ad esso associato o è birazionale sulla sua immagine o è di grado 2 su una superficie cubica con 4 punti doppi di $\boldsymbol{P}^{3}$ (cubica di Cayley) o è di grado 4 su $\boldsymbol{P}^{2}$. Nella terza sezione viene provato che se invece $D$ ha due punti base - pur soddisfacendo le altre ipotesi appena elencate - la mappa razionale $\varphi_{D}$ ad esso associata è di grado 2 o su $\boldsymbol{P}^{2}$ o su una superficie proiettivamente equivalente a una superficie $F_{n, r}$ con $n \leqslant 2$. Vengono inoltre determinate, in quest'ultimo caso, la classe di equivalenza lineare e le singolarità della curva di ramificazione di $\varphi_{D}$. (Il caso in cui l'immagine di $\varphi_{D}$ sia $\boldsymbol{P}^{2}$ è completamente trattato in ENRIQUES [1]).

Infine nella quarta sezione viene calcolata la dimensione dello spazio dei moduli delle superficie birazionalmente equivalenti a superficie di Enriques ottenibili come rivestimenti doppi di superficie $F_{n}$.

Le curve e superficie che considereremo saranno tutte definite su $C$. Se $X$ è uno schema proiettivo complesso e $\mathcal{F}$ un $o_{x}$-modulo indicheremo con $h^{i}(\mathcal{F})$ la dimensione di $H^{i}(X, \mathscr{F})$ come spazio vettoriale su $C$ e porremo $\chi(\mathscr{F})=\sum_{i \geqslant 0}(-1)^{i} h^{i}(\widetilde{F})$; se $D$ è un divisore su $X$ scriveremo anche $h^{i}(D)$ e $\chi(D)$ in luogo di $h^{i}\left(o_{X}(D)\right)$ e $\chi\left(o_{x}(D)\right)$ rispettivamente; $|D|$ sarà l'insieme dei divisori effettivi linearmente equivalenti a $D$ munito, se $|D| \neq \emptyset$, della consueta struttura di spazio proiettivo complesso; $\operatorname{dim}|D|=$ $=h^{0}(D)-1$ sarà la dịmensione di tale spazio; con $\equiv$ denoteremo l'equivalenza lineare tra divisori. Se $X$ è una varietà complessa proiettiva non singolare indicheremo con $K_{X}$ un suo divisore canonico. Se $D$ è un divisore effettivo su una superficie $X$ il genere aritmetico di $D$, denotato con $P_{a}(D)$, sarà l'intero $h^{1}\left(o_{D}\right)$. Converremo inoltre

(*) Entrata in Redazione il 21 dicembre 1979 
di dire che una trasformazione razionale è birazionale o di grado $n$ se lo è sulla sua immagine.

Per superficie di Enriques intenderemo ogni superficie proiettiva irriducibile non singolare $X$ tale che $h^{2}\left(o_{X}\right)=h^{1}\left(o_{X}\right)=0$ e $2 K_{X} \equiv 0$.

Ricordiamo che su una tale superficie l'equivalenza algebrica di divisori coincide con l'equivalenza lineare. In particolare un sistema lineare senza parti fisse su una superficie di Enriques o è irriducibile o è composto con un fascio lineare.

Vorrei ringraziare il prof. A. BeAUvILLE con il quale ho discusso questa ricerca.

1. - In questa sezione $X$ sarà sempre una fissata superficie di Enriques e scriveremo $K$ in luogo di $K_{X}$. Sia $D$ un divisore su $X$; il Riemann-Roch assume la forma:

$$
\chi(D)=\frac{D^{2}}{2}+1
$$

Se $D \geqslant 0$ da $h^{2}(D)=h^{0}\left(K_{X}-D\right)$ si ricava $h^{2}(D)=0$ e se $D=C$ curva irriducibile dalla successione esatta di cosmologia associata a $0 \rightarrow o_{X}(-C) \rightarrow o_{X} \rightarrow o_{C} \rightarrow 0$ si ricava

$$
h^{1}(-C)=h^{1}(K+C)=0 \quad \text { e } \quad P_{a}(C)=\frac{C^{2}}{2}+1 .
$$

Se $D \geqslant 0$ e $D$ è isolato - ossia $h^{0}(D)=1$ - dovrà aversi $D^{2} \leqslant 0$ e in particolare se $C$ è una curva irriducibile isolata su $X$ dovrà essere o $C^{a}=-2$ e quindi $C$ è una curva razionale non singolare, o $C^{2}=0$ e $O$ è o una curva ellittica non singolare $o$ una curva razionale con un punto doppio. Viceversa se $C$ è una curva irriducibile di $X$ la successione di coomologia associata a $0 \rightarrow o_{X} \rightarrow o_{X}(C) \rightarrow o_{C}(C) \rightarrow 0$ mostra che se $C^{2}=-2 C$ è isolata e $h^{1}(C)=1$ mentre se $C^{2}=0 O$ è isolata o varia in un fascio a seconda che $h^{0}\left(o_{C}(C)\right)\left(=h^{1}\left(o_{C}(C)\right)=h^{1}(C)\right)$ sia 0 o 1 ossia a seconda che $o_{C}(C)$ non sia o sia il fibrato triviale su $C$. In entrambi i casi si ha $o_{C}(C+K) \approx o_{C}$ dato che $h^{0}\left(o_{C}(C+K)\right)=h^{1}\left(o_{C}\right)=1$ e $(C+K) \cdot C=0$. Se è $C^{2}=0$ e $h^{0}(C)=2$ l'elemento generico di $|C|$ è una curva ellittica non singolare; inoltre la successione dI coomologia associata a $0 \rightarrow o_{X}(n C) \rightarrow o_{X}((n+1) C) \rightarrow o_{C} \rightarrow 0$ mostra che $h^{0}((n+$ $+1) \leqslant h^{0}(n C)+1$ e quindi che $h^{0}(n C)=n+1, h^{1}(n C)=n, \forall n \geqslant 0$ e che ogni $D \in|n C|$ si scrive $D=C_{1}+C_{2}+\ldots+C_{n}$ con $C_{i} \in|C|$. Se invece è $C^{2}=0$ e $h^{0}(C)=1$ la successione di coomologia associata a $0 \rightarrow o_{X}(C) \rightarrow o_{X}(2 C) \rightarrow o_{C}(2 C) \rightarrow 0$, tenuto conto del fatto che $o_{C}(2 C) \approx o_{C}(2 C+2 K) \approx o_{\sigma}$, ci dice che $|2 C|$ è un fascio ovviamente irriducibile il cui elemento generico è una curva ellittica non singolare.

Sia ancora $C$ irriducibile, $C^{2}=0, h^{0}(C)=2$; poichè $h^{\mathrm{i}}(K+C)=h^{1}(-C)=0$ si ha $h^{0}(K+C)=1$. Sia $D \in|K+C|$; poichè $2 D \equiv 2 K+2 C \equiv 2 C$ si può scrivere $2 D=C_{1}+C_{2}$ con $C_{i} \in|C|$ e $C_{1} \neq C_{2}$ (altrimenti $\left.D \in|C|\right)$. Essendo i supporti di $C_{1}$ e $C_{2}$ disgiunti tra loro (è $C^{2}=0$ e se $C_{1}$ e $C_{2}$ avessero una componente in comune questa sarebbe una componente fissa di $|C|)$ si può scrivere $D=D_{1}+D_{2} \operatorname{con} 2 D_{1}=C_{1}$ e $2 D_{2}=C_{2} ; D_{1}$ e $D_{2}$ sono connessi al pari di $C_{1}$ e $C_{2}$. 
In particolare $E \cdot C=2 E C_{i}$ è un numero pari qualunque sia il divisore $E$ di $X$ :

Propostzrone 1.1. - Sia $D$ un divisore effettivo su $X$ tale che $|D|$ non abbia componenti fisse. Si ha:
a) se $D^{2}>0$
$|D|$ è irriducibile ed è $h^{1}(D)=0$,
b) se $D^{2}=0 \quad$ esistono un intero $r \geqslant 1$ e un fascio irriducibile $|C|$ tali che $|D|=r C$.

Prova. - Se $|D|$ non è irriducibile è composto con un fascio (lineare) irriducibile $|C|$; se $|D|=r C$ con $r>1$ si ha $h^{0}(D)=r+1 \geqslant r^{2} C^{2} / 2+1$ il che è possibile solo se $C^{2}=0$, essendo $C^{2}$ un numero non negativo pari. Supponiamo che sia $D^{2}>0$ e che $\varepsilon: \tilde{X} \rightarrow X$ sia il ricoprimento doppio non ramificato di $X$ tale che $\varepsilon^{*} K \equiv 0$. Se $\tilde{D}=\varepsilon^{*} D,|\tilde{D}|$ non ha parti fisse ed è $\tilde{D}^{2}>0$ : come prima se ne deduce che $|\tilde{D}|$ è irriducibile e quindi che $h^{1}(\tilde{D})=h^{1}(-\tilde{D})=0$. Il fatto che $H^{1}\left(X, o_{x}(D)\right)$ possa essere identificato al sottospazio di $H^{1}\left(\widetilde{X} o_{\tilde{X}}(\widetilde{D})\right)$ lasciato fisso dalla trasformazione lineare indotta su di esso dall'automorfismo di $X$ che scambia i fogli del ricoprimento implica che anche $h^{1}(D)=0$.

Sia ora $D$ un divisore effettivo e tale che $D^{2}>0$ e sia $F$ la parte fissa di $|D|$ per cui $|D|=\left|D^{\prime}\right|+F$ con $\left|D^{\prime}\right|$ senza parte fissa. Si ha ancora $D^{\prime 2}>0$ a meno che $D^{\prime}$ sia un fascio irriducibile. In effetti se $D^{\prime 2}=0$ si può scrivere $\left|D^{\prime}\right|=r O$ con $|O|$ fascio irriducibile e dato che $F^{2} \leqslant 0$ esisterà una componente irriducibile $G$ di $F$ tale che $C \cdot G>0$.

Essendo $h^{0}\left(D^{\prime}+C\right)=h^{0}\left(D^{\prime}\right)=r+1,(r C+G)^{2} / 2+1=r C \cdot G+G^{2} / 2+1$ e tenuto conto che $G G$ è un numero pari e che $G^{2} / 2+1=P_{a}(G) \geqslant 0$ si dovrà avere $r=1, G^{2}=-2 \quad C G=2$.

Se $G^{\prime}$ è un'altra componente di $F$ distinta da $G$ per il Riemann Roch deve essere $2 \geqslant\left(C+G+G^{\prime}\right)^{2}=2+G^{\prime 2}+2 C G^{\prime}+2 G G^{\prime}$. Se fosse $G^{\prime 2}=0$ s'avrebbe $G^{\prime}(C+G)=0$ il che contraddirebbe il teorema dell'indice dunque è $G^{\prime 2}=-2$ ed essendo $C G^{\prime}$ pari è anche $O G^{\prime}=0$ ossia $G^{\prime}$ è una componente di un elemento di $C$.

Osserviamo che se $|C|$ è un fascio irriducibile con $C^{2}=0$ e $G$ è una curva irriducibile tale che $G^{2}=-2 C \cdot G=2$ si ha $|O+G|=|C|+G$. Ciò risulta dalla considerazione della successione di coomologia associata a $0 \rightarrow o_{X}(C) \rightarrow o_{X}(C+G) \rightarrow o_{G} \rightarrow 0$ e dal fatto che se $C+G$ fosse irriducibile si avrebbe $h^{1}(C+G)=0$. Sia ora $D$ un sistema lineare privo di componenti fisse con $D^{2}>0$ e sia $C$ una curva irriducibile: si ha $|D+C|=|D|+C$ se e solo se $C^{2}=-2$ e $C D \leqslant 1$. In effetti la successione di coomologia associata a $0 \rightarrow o_{X}(D) \rightarrow o_{X}(D+C) \rightarrow o_{C}(D+C) \rightarrow 0$ mostra che se $C^{2}=-2$ si ha $h^{0}(D)=h^{0}(D+C)$ se e solo se $C D \leqslant 1$ e che se $C^{2}=0$ è $h^{0}(D)=$ $=h^{0}(D+C)$ solo se $C D=0$ ciò che però è-impossibile per il teorema dell'indice.

Sia infine $|D|$ un sistema lineare completo senza componenti fisse e con $D^{2}>0$ su $X=X^{(0)}$. Possiamo considerare una successione di scoppiamenti

$$
\alpha_{1} \circ \alpha_{2} \circ \ldots \circ \alpha_{r}: X=X^{(r)} \rightarrow X^{(r-1)} \rightarrow \ldots \rightarrow X^{(2)} \rightarrow X^{(1)} \rightarrow X^{(0)}=X
$$


tale che, se

$$
\left|D^{(0)}\right|=|D| \quad \text { e } \quad\left|D^{(i)}\right|=\alpha_{i}^{-1}\left(\left|D^{(i-1)}\right|\right), \quad \alpha_{i+1}: X^{(i+1)} \rightarrow X^{(i)}
$$

è lo scoppiamento di un punto $P_{i}$ che è un punto base di molteplicità $n_{i} \geqslant 1$ di $\left|D^{(t)}\right|$ e tale che $\left|D^{(r)}\right|=|\tilde{D}|$ è privo di punti base e quindi ha come elemento generico una curva irriducibile non singolare.

Ricordiamo che se $E_{i+1}$ è la curva di $X^{(i+1)}$ che si contrae a $P_{i}$ si ha:

$$
\alpha_{i+1}^{-1}\left(\left|D^{(i)}\right|\right)=\left\{\alpha_{i+1}^{*} S-n_{i} E_{i+1}|S \in| D^{(i)} \mid\right\}=\left|\alpha_{i+1}^{*} D^{(i)}-n_{i} E_{i+1}\right| .
$$

Abbiamo:

$$
\tilde{D}^{2}=D^{2}-\sum_{i=0}^{r-1} n_{i}^{2} \quad P_{a}(\tilde{D})=P_{a}(D)-\sum_{i=0}^{r-1} \frac{n_{i}\left(n_{i}-1\right)}{2}
$$

e dalla successione di coomologia associata a $0 \rightarrow o_{\tilde{X}} \rightarrow o_{\tilde{X}}(\tilde{D}) \rightarrow o_{\tilde{D}}(\tilde{D}) \rightarrow 0$ dato che $h^{1}\left(o_{\tilde{x}}\right)=h^{1}\left(o_{\tilde{X}}\right)=0$ si ricava:

$$
h^{0}\left(o_{\bar{D}}(\tilde{D})\right)=h^{0}(\tilde{D})-1=h^{0}(D)-1=\frac{D^{2}}{2} .
$$
Poichè si ha: $2 P_{a}(\tilde{D})-2=D^{2}-\sum_{i=0}^{r-1} n_{i}\left(n_{i}-1\right) \geqslant \tilde{D}^{2}$ il teorema di Clifford è ap-
plicabile e da esso segue che

$$
\frac{D^{2}}{2} \leqslant \frac{1}{2}\left(D^{2}-\sum_{i=0}^{r-1} n_{i}^{2}\right)+1 \quad \text { ossia che } \sum_{i=0}^{r-1} n_{i}^{2} \leqslant 2 .
$$

Risulta dunque che la curva generica di $|D|$ è non singolare e che $|D|$ ha al più due punti base eventualmente "infinitamente vicini".

Supponiamo che $|D|$ abbia almeno un punto base $P$; la serie lineare segata da $|D|$ su un suo membro irriducibile non singolare $C$ è una serie completa (come si vede dalla successione di coomologia associata e $\left.0 \rightarrow o_{X} \rightarrow o_{X}(D) \rightarrow o_{C}(D) \rightarrow 0\right)$ di grado $2 g-2$ e dimensione $g-2$, se $g=D^{2} / 2+1$ è il genere di $C$, che ha $P$ come punto fisso. Sia $|A|$ tale serie; per il Riemann-Roch su $C|A-P|$ è speciale e possiamo scrivere $K_{C}-A+P \equiv Q$ con $Q \in O$ e $Q \neq P$ (altrimenti $\left.|A|=\left|K_{C}\right|\right)$. Poichè è $2 K_{o} \equiv 2 A$ abbiamo $2 P \equiv 2 Q$. Dunque $C$ possiede una $g_{2}^{1}$, è cioè iperellittica, e $P$ e $Q$ sono punti doppi di tale $g_{2}^{1}$. Essendo $\left|K_{c}\right|=|(2 g-2) Q|$ possiamo scrivere

$$
|A-P|=\left|K_{C}-Q\right|=|(g-2) 2 Q+Q|=(g-2) 2 Q+Q .
$$

Dunque $Q$ è un'altro punto fisso di $A-$ e quindi di $|D|-$ e $|A-P-Q|$ è composta dalla $g_{2}^{1}$ di $C$. 
Abbiamo così dimostrato la seguente

Proposizione 1.2. - Sia $|D|$ senza parti fisse e con $D^{2}>0$; o $|D|$ non ha punti base o ne ha esattamente 2 nel qual caso il suo elemento generico è una curva non singolare iperellittica, i punti base sono punti doppi della $g_{2}^{1}$ appartenente a tale curva e la serie lineare completa segata da $D$ sulla curva, tolti i punti fissi, è compossta dalla $g_{2}^{1}$.

\section{2. - In questa sezione proveremo la seguente}

Proposiztone 2. - Sia $X$ una superficie di Enriques e sia $|D|$ un sistema lineare completo su $X$ privo di parti fisse e di punti base e con $D^{2}>0$. Sia $\varphi_{D}: X \rightarrow \boldsymbol{P}^{D^{2} / 2}$ il morfismo associato a $|D|$. Si ha che $0 \varphi_{D}$ è birazionale o $D^{2}=6$ e $\varphi_{D}$ è un morfismo di grado 2 la cui immagine è la cubica di Cayley - una cubica di $\boldsymbol{P}^{3}$ con 4 punti doppi - o $D^{2}=4$ e $\varphi_{D}$ è un morfismo di grado 4 la cui immaginè è $\boldsymbol{P}^{2}$.

Prova. - Sia $F=\varphi_{D}(X)$. Dovendo essere $\operatorname{deg} F \cdot \operatorname{deg} \varphi_{D}=D^{2}$ e $\operatorname{deg} F \geqslant$ $\geqslant \operatorname{codim} F+1=D^{2} / 2-1$ a priori sono possibili soltanto $i$ seguenti 4 casi: $\left.a\right) \varphi_{D}$ è birazionale $b) \operatorname{deg} \varphi_{D}=2$ e $\operatorname{deg} F=D^{2} / 2 \quad$ o) $\operatorname{deg} \varphi_{D}=3, D^{2}=6, \operatorname{deg} F=2$ d) $\operatorname{deg} \varphi_{D}=4 D^{2}=4 F=\boldsymbol{P}^{2}$. Sia $C$ un elemento irriducibile non singolare di $|D|$ e sia $\Gamma=\varphi_{D}(C)$. Se deg $\varphi_{D} \geqslant 2 \Gamma$ è una curva di un $\boldsymbol{P}^{D^{2} / 2-1}$ di grado $\leqslant D^{2} / 2$ che non giace in alcun $\boldsymbol{P}^{D^{2} / 2-1}$ per cui è o una curva ellittica non singolare o una curva razionale.

La serie lineare segata da $|D|$ su $C$ è completa e senza punti fissi: denotiamola con $|A|$. Se $g=P_{a}(C)=D^{2} / 2+1$ è $\operatorname{deg} A=2 g-2$ e $\operatorname{dim}|A|=g-2$. Ora se $\operatorname{deg} \varphi_{D}=2$ e $\Gamma$ fosse razionale $O$ sarebbe iperellittica e $|A|$ sarebbe composta con la $g_{2}^{1}$ di $C$ il che è impossibile visto che $|A|$ non ha punti fissi e $\operatorname{dim}|A|=g-2$.

Se invece fosse $\operatorname{deg} \varphi_{D}=3$ (e quindi $D^{2}=6$ e $P_{a}(C)=4$ ) $\Gamma$ sarebbe una conica piana e potremmo scrivere $|A|=\left|2 A^{\prime}\right|$ con $\left|A^{\prime}\right|$ una $g_{3}^{1}$ senza punti fissi. Per il RiemannRoch si potrebbe anche scrivere $K_{C} \equiv A^{\prime}+A^{\prime \prime}$ con $\left|A^{\prime \prime}\right|$ una $g_{3}^{1}$ diversa da $\left|A^{\prime}\right|$ - dato che $\left|K_{C}\right| \neq|A|-$.

Da $2 K_{0} \equiv 2 A \equiv 4 A^{\prime} \equiv 2 A^{\prime}+2 A^{\prime \prime}$ ricaveremmo $2 A^{\prime} \equiv 2 A^{\prime \prime} \equiv A$ e quindi $A^{\prime} \equiv A^{\prime \prime}$.

Abbiamo così mostrato che il caso $c$ ) non si verifica e che nel caso $b$ ) la sezione iperpiana generica di $F$ è una curva ellittica non singolare.

Supponiamo che sia $\operatorname{deg} \varphi_{D}=2$. La trasformazione birazionale $\sigma: X \rightarrow X$ distinta dall'identità che rende commutativo il diagramma

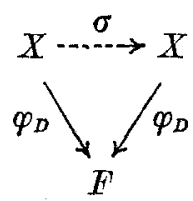

essendo $X$ non rigata e minimale è un automorfismo. 
Sia $\pi: X \rightarrow X / \sigma$ il quoziente di $X$ per l'azione di $\{\sigma, 1\} ; X / \sigma$ è una superficie normale la cui singolarità sono punti doppi ordinari immagini, tramite $\pi$, dei punti fissi isolati dall'azione di $\{\sigma, 1\}$ su $X$. Siano $P_{1} \ldots P_{r}$ tali punti e sia $\alpha: \tilde{X} \rightarrow X$ la superficie ottenuta da $X$ scoppiando $P_{1} \ldots P_{r}$. Se $\tilde{\sigma}: \tilde{X} \rightarrow \tilde{X}$ è l'automorfismo di $\tilde{X}$ indotto da $\sigma$ e $\tilde{\pi}: \tilde{X} \rightarrow \tilde{X} / \tilde{\sigma}=\tilde{S}$ il quoziente di $\tilde{X}$ per l'azione di $\{\tilde{\sigma}, 1\}$, $\tilde{S}$ è non singolare. Siano $E_{1} \ldots E_{r}$ le curve di $X$ che si contraggono a $P_{1} \ldots P_{r}$ rispettivamente; poniamo $C_{i}=\tilde{\pi}_{*}\left(E_{i}\right)$ cosicchè $\tilde{\pi}^{*}\left(O_{i}\right)=2 E_{i}$. Dato che $E_{i}^{2}=-1$ si ha $C_{i}^{2}=-2$ e dato che le $C_{i}$ sono razionali non singolari è $C_{i} K_{\tilde{S}}=0$. Sia $\Delta$ la curva luogo di ramificazione di $\tilde{\pi}$ e sia $\delta$ il divisore di $\tilde{S}$ canonicamente associato al rivestimento doppio $\tilde{\pi}: \tilde{X} \rightarrow \tilde{S}$ tale che $\Delta \in|2 \delta|$.

Abbiamo:

$$
K_{\tilde{\Sigma}} \equiv \tilde{\pi}^{*}\left(\ddot{K}_{\tilde{R}}+\delta\right) \equiv \alpha^{*} K_{X}+\sum_{i=1}^{r} E_{i}
$$

da cui si ricava

$$
2 \tilde{\pi}^{*}\left(K_{\bar{s}}+\delta\right) \equiv 2 \sum_{i=1}^{r} E_{i} \equiv \tilde{\pi}^{*}\left(\sum_{i=1} C_{i}\right)
$$

Poichè $\tilde{\pi}^{*}: \tilde{X} \rightarrow \tilde{S}$ è un rivestimento doppio ramificato $\tilde{\pi}^{*}: \operatorname{Pic} \tilde{S} \rightarrow \operatorname{Pic} \tilde{X}$ è iniettivo e si ha: $2\left(K_{\tilde{s}}+\delta\right) \equiv \sum_{i=1}^{r} C_{i}$

Da

$$
\chi\left(o_{\tilde{X}}\right)=1=2 \chi\left(o_{\tilde{s}}\right)+\frac{1}{2} \delta\left(\delta+K_{\tilde{s}}\right)=2+\frac{1}{8}\left(\sum_{i=1}^{r} C_{i}\right)\left(\sum_{i=1}^{r} o_{i}-2 K_{\tilde{s}}\right)=2-\frac{r}{4}
$$

ricaviamo infine $r=4$.

Poniamo $D^{2} / 2=d . F$, essendo una superficie di grado $d$ in $\boldsymbol{P}^{d}$ che non giace in alcun $\boldsymbol{P}^{a^{-1}}$, è a priori, come noto (NAGATA [1]), proiettivamente equivalente a uno dei seguenti tipi di superficie:

1) proiezioni di $\boldsymbol{P}^{d}$ di superficie di grado $d$ in $\boldsymbol{P}^{d+1}$ non giacenti in alcun $\boldsymbol{P}^{d}$ da un punto esterno ad esse,

2) immagini birazionali di $\boldsymbol{P}^{2}$ tramite un sistema lineare di cubiche, generi. camente non singolari, con al più 6 punti base,

3) trasformate di Veronese in $\boldsymbol{P}^{8}$ in quadriche anche singolari in $\boldsymbol{P}^{\mathbf{3}}$,

4) coni su curve ellittiche non singolari.

$F$ non può essere di tipo 4) dato che non esistono morfismi suriettivi di $X$ su superficie irregolari nè di tipo 1) dato che le sezioni iperpiane di superficie di questo tipo sono curve razionali. Abbiamo appena visto che $X / \sigma$ ha esattamente 4 punti doppi ordinari: ciò implica che $F$ ha almeno 4 punti singolari ed esclude che $F$ possa essere di tipo 3 ). 
Possiamo perciò supporre che $F$ sia di tipo 2). Sia $L$ il sistema lineare di cubiche piane tale che, se $\varphi_{L}$ è la trasformazione razionale di $\boldsymbol{P}^{2}$ ad esso associato, sia $\varphi_{L}\left(\boldsymbol{P}^{2}\right)=F$. Sia $\alpha: Z \rightarrow \boldsymbol{P}^{2}$ ottenuta scoppiando i punti fissi di $L$ (anche quelli "infinitamente vicini", come nella prova della Proposizione 1.2) per modo che $\alpha^{-1} L$ sia senza punti fissi e si abbia un diagramma commutativo

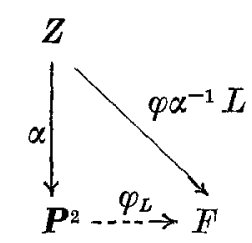

Poichè $F^{\prime}$ ha almeno 4 punti singolari esistono 4 curve irriducibili $C_{1} C_{2} C_{3}$ e $C_{4}$ di $Z$ tali che $C_{i}^{2} \leqslant-2$ e che vengono contratte a un punto da $\varphi_{\alpha^{-1} L} . \alpha\left(C_{i}\right)$, dovendo essere una componente fissa degli elementi di un iperpiano di $L$, è o una retta o una conica non singolare. Se $\alpha\left(O_{i}\right)$ fosse una conica non singolare, per essere $C_{i}^{2} \leqslant-2, L$ dovrebbe avere 6 punti base (contando anche quelli «infinitamente vicini») e $\alpha\left(C_{i}\right)$ dovrebbe passare per essi. E facile convincersi che in tal caso si avrebbe $\sigma_{j}^{2} \geqslant-1$ $\forall j \neq i$. Dunque $\alpha\left(C_{i}\right)$ è uro retta $\forall i$ e passa per 3 punti base del fascio (a priori anche "infinitamente vicini»). Ciò implica evidentemente che $L$ ha 6 punti base distinti che sono i vertici di un quadrilatero completo.

La prova della proposizione 2 è così terminata.

Vogliamo ora mostrare, usando una costruzione dovuta a A. BEAUVILLE, che esistono superfici di Enriques $X$ muniti di un sistema lineare completo $|D|$ senza componenti fisse o punti base tale che $\varphi_{D}(X)$ sia la cubica di Cayley.

Siano $F^{\prime} \subseteq \boldsymbol{P}^{3}, \alpha: Z \rightarrow \boldsymbol{P}^{2}$ e $C_{i}(i=1, \ldots, 4)$ come sopra con $L$ sistema lineare di tutte le cubiche passanti per i vertici di un quadrilatero completo. Sia $H$ una sezione iperpiana di $F$ : si ha $K_{Z} \equiv-\varphi_{\alpha^{-1} L}^{*}(H)$. Sia $Q$ una sezione di $F$ con una quadrica di $\boldsymbol{P}^{3}$ tale che $\varphi_{x^{-1} I_{L}}^{*}(Q)$ sia irriducibile e non singolare e sia $\tilde{\varrho}: \tilde{X} \rightarrow Z$ il rivestimento doppio di $Z$ ramificato lungo $A=\varphi_{\alpha^{-1} L}^{*}(Q)+\sum_{i=1}^{4} C_{i}$ (si noti che se $E_{1} \ldots E_{6}$ sono le curve eccezionali di $Z$ e $l$ è una retta di $\boldsymbol{P}^{2}$ si ha $\sum_{i=1}^{4} C_{i} \equiv 2\left(\alpha^{*}(2 l)-\sum_{i=1}^{6} E_{i}\right)$ per cui $\Delta \in|2 \delta|$ con $\delta \equiv \varphi_{\alpha^{-1} L}^{*}(H)+\left(\alpha^{*}(2 l)-\sum_{i=1}^{6} t_{i}\right)$.

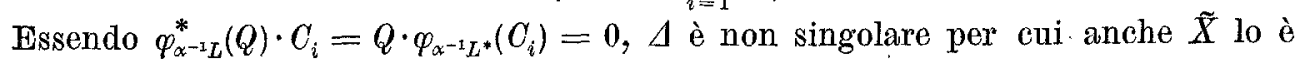
e si ha:

$$
\begin{aligned}
& \chi\left(o_{\tilde{X}}\right)=2 \chi\left(o_{Z}\right)+\frac{1}{2} \delta\left(\delta+Z_{Z}\right)=2+\frac{1}{8} \Delta\left(\Delta+2 K_{Z}\right)=1 \\
& 2 K_{\tilde{x}} \equiv \tilde{\varrho}^{*}\left(2 K_{Z}+\Delta\right) \equiv \tilde{\varrho}^{*}\left(\sum_{i=1}^{4} C_{i}\right) .
\end{aligned}
$$

Essendo le $C_{i}$ componenti del luogo di ramificazione si può scrivere $\tilde{g}^{*} C_{i}=2 G$ ed è $G_{i}^{2}=-1$. Sia $\varepsilon: \tilde{X} \rightarrow X$ la superficie ottenuta da $\tilde{X}$ contraendo $G_{1} \ldots G_{4}$ : 
Poichè $2 K_{\tilde{x}} \equiv \varepsilon^{*}\left(2 K_{X}\right)+\sum_{i=1}^{4} 2 G_{i} \equiv \tilde{\varrho}\left(\sum_{i=1}^{4} G_{i}\right) \equiv \sum_{i=1}^{4} 2 G_{i}$ ne segue $2 K_{x} \equiv 0 . \quad X$ è perciò una superficie di Enriques e $\tilde{\varrho}$ induce un morfismo di grado $2 \varrho: X \rightarrow F$. Sia $D \equiv \varrho^{*}(H)$; è $D^{2}=6, h^{0}(D)=4$ per cui $\varrho=\varphi_{D}$.

3. - Sia $D$ un sistema lineare completo su una superficie di Enriques $X$ privo di componenti fisse, con $D^{2}>0$ e con due punti base $P_{1}$ e $P_{2}$. Sia $\varepsilon: \hat{X} \rightarrow X$ lo scoppiamento di $P_{1}$ e $P_{2}$ e siano $E_{1}$ ed $E_{2}$ le curve eccezionali di $X$ che si contraggono a $P_{1}$ e $P_{2}$ rispettivamente; se $\hat{D} \equiv \varepsilon^{*} D-E_{1}-E_{2}|\hat{D}|$ non ha punti base, è $\hat{D}^{2}=D^{2}-2, D E_{i}=1$ e si ha un diagramma commutativo

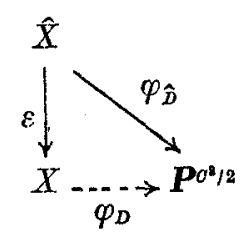

Sappiamo che ogni curva irriducibile non singolare $C$ di $|D|$ è iperellittica e che la trasformazione razionale $\varphi_{D}$ ristretta a $C$ è data da una serie lineare composta con la $g_{2}^{\mathbf{1}}$ di $C . \varphi_{D}$ e $\varphi_{\hat{D}}$ hanno pertanto grado 2 e $F=\varphi_{\hat{D}}(\hat{X})$ è una superficie di grado $D^{2} / 2-1$ in $\boldsymbol{P}^{\mathbb{R}^{2} / 2}$. Scriveremo d'ora in poi $\varphi$ in luogo di $\varphi_{\hat{D}}$ e porremo $D^{2} / 2=d$. Assumeremo $d \geqslant 3$. (Il caso $d=2$ come ben noto è trattato in Enriques [1]). Poichè $P_{1}$ e $P_{2}$ sono puñti doppi della $g_{2}^{2}$ appartenente all'elemento generico $C$ di $|D| \varphi$ è ramificato Iungo $E_{1}$ ed $E_{2}$. Inoltre se $H$ è una sezione iperpiana di $F$ si ha $\varphi_{*}\left(E_{i}\right) \cdot H=E_{i} \cdot \varphi^{*}(H)=E_{i} \hat{D}=1$ per cui $\varphi_{*}\left(E_{i}\right)$ è una retta di $\boldsymbol{P}^{d}$. È noto che una superficie di grado $d-1$ in $\boldsymbol{P}^{d}$ che non giace in alcun $\boldsymbol{P}^{d-1}$ è proiettivamente equivalente a una superficie $\boldsymbol{F}_{n, r}$ con $n+2 r+1=d$ o alla superficie di Veronese $V_{4} \subseteq \boldsymbol{P}^{5}$.

Poichè la superficie di Veronese non contiene rette $F$ deve essere proiettivamente equivalente a una superficie $F_{n, r}$. Ricordiamo che la superficie $F_{n}(n \geqslant 0)$ è il fibrato con base $\boldsymbol{P}^{1}$ e fibra $\boldsymbol{P}^{1}$ ottenuto incollando i due fibrati banali $\boldsymbol{P}^{1}-\{\infty\} \times \boldsymbol{P}^{1} \rightarrow \boldsymbol{P}^{1}-$ $-\{\infty\}, \boldsymbol{P}^{1}-\{0\} \times \boldsymbol{P}^{1} \rightarrow \boldsymbol{P}^{1}-\{0\}$ tramite $\left(t ; X_{0}: X_{1}\right) \mapsto\left(1 / t ; t^{n} X_{0}: X_{1}\right)$. Sia $B$ l'jmmagine della sezione $t \mapsto(t ; 0: 1) \infty \mapsto(\infty ; 0: 1)$ e $A$ una qualsiasi fibra. Pic $\mathbb{F}_{n}$ è il gruppo abeliano libero generato dalle classi di $A$ e $B$ e si ha $A^{2}=0, A B=1$, $B^{2}=-n, K_{F_{n}} \equiv-(n+2) A-2 B$.

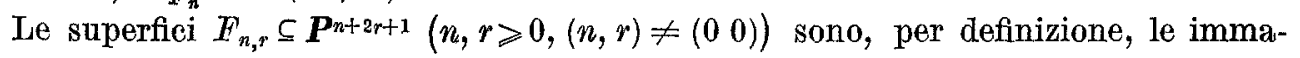
gini di $F_{n}$ per i morfismi $\varphi_{n, r}$ associati ai sistemi lineari $|(n+r) A+B|$; tali sistemi lineari sono privi di componenti fisse e punti base e sono irriducibili $\forall r \geqslant 0$, inoltre se $r>0 \varphi_{n, r}$ è un isomorfismo mentre $F_{n, 0}$ è un cono con vertice $\varphi_{n, 0}(B)=V$ e $\varphi_{n, 0}$ induce un isomorfismo tra $F_{n}-B$ e $F_{n, 0}^{\prime}-V$ che porta il sistema lineare $|A|$ nelle generatrici di $F_{n, 0}$.

$\mathbf{E}$ facile verificare che le uniche rette contenute in una $F_{n, r}$ sono le immagini tramite $\varphi_{n, r}$ degli elementi del sistema $|A|$ eccettuato il caso in cui $r=1$ nel qual caso anche le immagini del sistema $|B|$ sono rette. 
Torniamo ora a considerare il morfismo $\varphi$ : vogliamo mostrare che se $F$ è proiettivamente equivalente a una $F_{n, 0}$ esiste una curva $C$ di $X$ tale che il sistema $|D+C|$ è senza parti fisse, ha ancora $P_{1}$ e $P_{2}$ come punti base e dà luogo a un diagramma commutativo

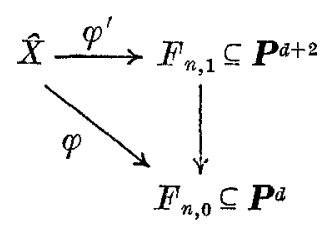

$\varphi^{\prime}$ essendo il morfismo associato al sistema lineare $\left|\varepsilon^{*}(D+C)-E_{1}-E_{2}\right|$.

Il sistema algebrico $\left\{\varphi^{*}(G)\right\}$, al variare di $G$ tra le generatrici di $F_{n, 0}$, è un sistema lineare, dato che $\hat{X}$ è regolare, la cui parte variabile è irriducibile dato che altrimenti sarebbe composta con un fascio di curve razionali e $\hat{X}$ sarebbe razionale. Sia $\hat{C}$ un elemento irriducibile di tale parte variabile e sia $H$ una sezione iperpiana di $F_{n, 0}$ attraverso il suo vertice $V$. Considerando $H$ come divisore di Weil $\left(F_{n, 0}\right.$ è normale $)$ possiamo scrivere $H=G_{1}+\ldots+G_{d-1}, G_{i}$ essendo una generatrice, e quindi $\varphi^{*}(H) \equiv$ $\equiv(d-1) \hat{C}+\sum n_{i} F_{i}$ con $n_{i} \geqslant 0$ e $\varphi\left(F_{i}\right)=V$. Gli $n_{i}$ non possono essere tutti nulli dato che altrimenti si avrebbe $1=\hat{D} \cdot T_{i}=\varphi^{*}(H) \cdot E_{i}=(d-1) \hat{C} \cdot E_{i}$.

Se $H^{\prime}$ è una sezione iperpiana di $F_{n, 0}$ che non passa per il vertice possiamo scrivere $\hat{D} \cdot \hat{C}=H^{\prime} \cdot \varphi_{*}(\hat{C})=H^{\prime} \cdot 2 G=2=(d-1) \hat{C}^{2}+\sum n_{i} F_{i} \cdot \hat{C}$.

Dato che $d \geqslant 3$ e dato che $(d-1) \hat{C}+\sum n_{i} F_{i}$ è connesso deve essere $\hat{C}_{2}=0$; è inoltre $\hat{C} \cdot E_{i}=0$ dato che si può scrivere $\hat{C} \equiv E_{i}+M_{i}$ con $M_{i} \geqslant 0$.

Esiste dunque una curva irriducibile $C$ che non passa nè per $P_{1}$ nè per $P_{2}$ tale che $\varepsilon^{*} C=\hat{C}$ e che $C^{2}=0, C D=2$; per costruzione $C$ non è isolata e quindi $|C|$ è un fascio. Il sistema lineare $\left|D^{\prime}\right|=|D+C|$ è evidentemente privo di parti fisse: si ha $D^{\prime 2}=D^{2}+4, h^{0}\left(D^{\prime}\right)=d+3$.

Consideriamo la serie lineare segata da $D^{\prime}$ su un elemento irriducibile non singolare $\Gamma \in|D|$. La successione di coomologia associata an $0 \rightarrow o_{X}(C) \rightarrow o_{X}\left(D^{\prime}\right) \rightarrow o_{\Gamma}\left(D^{\prime}\right) \rightarrow 0$ mostra che tale serie ha dimensione $d$; d'altra parte la serie segata da $|D|$ su $\Gamma$ ha dimensione $d-1$ e la serie segata da $C$ su $T$ è una $g_{2}^{1}$ come si vede dalla successione di coomologia associata a $0 \rightarrow o_{X}(C-D) \rightarrow o_{X}(C) \rightarrow o_{\Gamma}(C) \rightarrow 0$ (non può essere $C-D \equiv L$ con $L \geqslant 0$ poichè altrimenti sarebbe $D(C-D)=2-2 d \geqslant 0)$. In definitiva ogni elemento della serie segata da $\left|D^{\prime}\right|$ su $\Gamma$ si può scrivere come somma di un elemento della serie segata da $|D|$ su e di uno della serie segata da $|C|$ su $\Gamma$. In particolare la serie segata da $\left|D^{\prime}\right|$ su $\Gamma$ ha $P_{1}$ e $P_{2}$ come punti fissi il che equivale a dire che $P_{1}$ e $P_{2}$ sono punti base di $\left|D^{\prime}\right|$. Sia $\left|\hat{D}^{\prime}\right|=\left|\varepsilon^{*} D^{\prime}-E_{1}-E_{2}\right|$ e $\varphi^{\prime}$ il morfismo ad esso associato. I'immagine di $\varphi^{\prime}$ è a priori una superficie $F_{m, r}$ con $m+2 r+1=d+2$. Osserviamo che $\left|\hat{D}^{\prime}-(d+1) \hat{C}\right|=\left|\sum n_{i} F_{i}-\hat{C}\right|=\emptyset$ dato che $\hat{D}\left(\sum n_{i} F_{i}\right)=0$ e $\hat{D} \hat{C}=2$, mentre $\left|\hat{D}^{\prime}-\partial \hat{C}\right|=\left|\sum n_{i} F_{i}\right| \neq \emptyset$. Ciò equivale a dire che esistono sezioni iperpiane di $F_{m, r}$ che contengono $d$ elementi di $\varphi_{m, r}(|A|)$ mentre non ne esistono che ne contengono $d+1$. Per note proprietà delle superficie $F_{m, r}$ deve essere $m+r=d$ e quindi $r=1$. 
Osserviamo che la costruzione di $\left|D^{\prime}\right|$ a partire da $|D|$ può essere fatta, in modo identico, nel caso in cui $F$ sia proiettivamente equivalente a una qualsiasi $F_{n, r}$ Supporremmo da ora in poi che l'immagine $F$ di $\varphi$ sia proiettivamente equivalente a una $F_{n, r}$ con $r \geqslant 1$ e scriveremo $A$ e $B$ in luogo di $\varphi_{n, r}(A)$ e $\varphi_{n, r}(B)$. Facciamo ora vedere che si ha $\varphi^{*}\left(E_{i}\right) \in|A|$ - salvo scambiare nel caso $n=0, r=1$ i ruoli d'altronde simmetrici di $A$ e $B$.

In effetti $\varphi^{*}(A)\left(\varphi^{*}(A)+K_{\tilde{x}}\right)=\varphi^{*}(A)\left(E_{i}+E_{2}\right)=A\left(\varphi_{*}\left(E_{1}\right)+\varphi_{*}\left(E_{2}\right)\right)$ deve essere un numero pari per cui o $\varphi_{*}\left(E_{i}\right) \in|A|(i=1,2)$ o $\varphi_{*}\left(E_{i}\right) \in|B|(i=1,2)$ e $r=1$ (ricordiamo che $\varphi_{*}\left(E_{i}\right)$ è una retta di $\boldsymbol{P}^{a}$ ). Se $n>0 B$ è isolata ed essendo $\varphi$ di grado 2 e ramificato lungo $E_{1}$ ed $E_{2}$ non è possibile avere $\varphi_{*}\left(E_{1}\right)=\varphi_{*}\left(E_{2}\right)=B$.

Sia $C$ la curva di $F$ luogo della ramificazione di $\varphi$ e sia $C \equiv a A+b B$; vogliamo determinare $a$ e $b$. Sia $H \equiv(n+r) A+B$ una sezione iperpiana irriducibile e non singolare di $F$ tale che $\phi^{*} H$ sia irriducibile e non singolare e sia $A$ tale che $\varphi^{*} A$. sia irriducibile e non singolare. 亡̀ $2 P_{a}(A)-2=P_{a}(H)-2=-2$ e $2 P_{a}\left(\varphi^{*}(A)\right)-$ $-2=\varphi^{*}(A)\left(\varphi^{*}(A)+K_{\hat{X}}\right)=02 P_{a}\left(\varphi^{*} H\right)-2=\varphi^{*}(H)\left(\varphi^{*}(H)+K_{\hat{x}}\right)=2+2 n+4 r$.

La formula di Hurwitz applicata alle restrizioni di $\varphi$ a $\varphi^{*} A$ e $\varphi^{*} H$ rispettivamente da: $C \cdot A=b=4, \quad C \cdot H=a+b r=6+2 n+4 r$. Abbiamo perciò $O \in|(2 n+6) A+4 B|=\left|-2 K_{F_{n, r}}+2 A\right|$.

Allo scopo di studiare le singolarità di $C$ considereremo la situazione seguente: $S^{(0)}=S$ è una superficie proiettiva non singolare, $\pi^{(h)}: S^{(h)} \rightarrow S^{(h-1)}$ per $h \geqslant 1$ è lo scoppiamento dei punti $P_{J_{1} \ldots J_{h}}$ di $S^{(h-1)}$ (ometteremo di precisare la variabilità degli indici: s'intende che essi variano compatibilmente con le assunzioni fatte), $E_{J_{1} \ldots J_{h}}$ è la curva eccezionale di $S^{(k)}$ che si contrae a $P_{J_{1} \ldots J_{h}} . E_{J_{1} \ldots J_{h}}$ indicherà anche la trasformata propria di $E_{J_{1} \ldots J_{h}}$ in ognuna delle $S^{(k)} \operatorname{con} k \geqslant h$ e porremo $\pi=\pi^{(1)} \circ \pi^{(2)} \ldots \circ \pi^{(h)}$ qualunque sia $h$ confidando che tali ambiguità non generino confusione.

Supporremo che sia $P_{J_{1} \ldots J_{h}} \in E_{J_{1} \ldots J_{h-1}}$ e, per $1 \leqslant h \leqslant k-1$, l'intero $\varepsilon_{J_{1} \ldots J_{h} \ldots J_{k}}^{J_{1} \ldots J_{h}}$ sarà definito così : $\varepsilon_{J_{1} \ldots J_{h}}^{J_{1} \ldots J_{k}}=0$ se $P_{J_{1} \ldots J_{h}} \notin E_{J_{1} \ldots J_{h}}, \varepsilon_{J_{1} \ldots J_{h}}^{J_{1} \ldots J_{k}}=1$ se $P_{J_{1} \ldots J_{k}} \in E_{J_{1} \ldots J_{h}}$ (per ipotesi è $\varepsilon_{J_{1} \ldots J_{k}}^{J_{1} \ldots J_{k-1}}=1$ ed è $\varepsilon_{J_{1} . . . J_{h}}^{J_{1} \ldots J_{k}}=1$ al più per un solo valore di $\left.h \neq k-1\right)$.

Abbiamo

$$
K_{S^{(k)}}=\pi^{*}\left(K_{S}\right)+\sum_{E_{1}} c_{O_{1}} E_{J_{1}}+\sum_{J_{1} J_{2}} c_{J_{1} J_{1}} E_{J_{1} J_{2}} \ldots+\sum_{J_{1} \ldots J_{k}} c_{J_{1} \ldots J_{k}} E_{J_{\perp} \ldots J_{k}}
$$

con i $o_{J_{1} \ldots J_{h}}$ determinati ricorsivamente da:

$$
o_{J_{1}}=1 \quad \forall J_{1}, \quad e_{J_{1} \ldots J_{h}}=1+c_{J_{1}} \varepsilon_{J_{1} \ldots J_{h}}^{J_{1}}+c_{J_{1} J_{2}} \varepsilon_{J_{1} \ldots J_{k}}^{J_{1} J_{2}}+\ldots+c_{J_{1} \ldots J_{h-1}} \varepsilon_{J_{1} \ldots J_{h}}^{J_{1} \ldots J_{h-1}}
$$

Sia $D$ un divisore effettivo di $S$ e sia $n_{J_{1} \ldots J_{b}}$ la molteplicità di $P_{J_{1} \ldots J_{h}}$ per la trasformata propria di $D$ in $\mathcal{S}^{(h)}$. Se $D^{(h)}=\pi^{-1} D$ è tale trasformata si ha:

$$
D^{(k)}=\pi^{*}(D)-\sum_{J_{1}} d_{J_{1}} E_{J_{1}}-\sum_{J_{1} J_{\mathrm{a}}} d_{J_{1} J_{3}} E_{J_{1} J_{2}} \ldots-\sum_{J_{1} \ldots J_{k}} d_{J_{1} \ldots J_{k}} E_{J_{1} \ldots J_{k}}
$$


con i $d_{J_{1} \ldots J_{n}}$ determinati ricorsivamente da:

$d_{u_{1}}=n_{J_{1}} \quad \forall J_{1}, \quad d_{J_{1} \ldots J_{h}}=n_{J_{1} \ldots J_{h}}+d_{J_{1}} \varepsilon_{J_{1} \ldots J_{h}}^{J_{1}}+d_{J_{1} J_{2}} \varepsilon_{J_{1} J_{2} \ldots J_{h}}^{J_{1} J_{2}}+\ldots$

$$
\ldots+d_{J_{1} \ldots J_{h-1}} \varepsilon_{J_{1} \ldots J_{h}}^{J_{1} \ldots J_{h-1}} .
$$

Sia $D$ privo di componenti multiple: è possibile trovare una successione $\tilde{S}=S^{(k)} \rightarrow$ $\rightarrow S^{(k-1)} \rightarrow \ldots \rightarrow S^{(1)} \rightarrow S^{(0)}$ tale che, se $\delta_{J_{1} \ldots \nu n}(1 \leqslant h \leqslant k)$ è un intero che vale 0 o 1 determinato dalla condizione che $d_{J_{1} \ldots J_{h}}-\delta_{J_{1} \ldots J_{h}}$ sia un numero pari, la curva:

$$
\widetilde{D}=D^{(k)}+\sum_{J_{1}} \delta_{S} E_{J_{1}}+\sum_{J_{1} J_{2}} \delta_{J_{1} J_{2}} E_{J_{1} J_{2}} \ldots+\sum_{J_{1} \ldots J_{k}} \delta_{J_{1} \ldots J_{k}} E_{J_{1} \ldots J_{k}}
$$

sia non singolare.

Ciò è pressochè evidente se si considera che effettuando opportuni scoppiamenti si può successivamente fare sì che la trasformata propria di $D$ sia non singolare, che tutte le curve $E_{J_{1} \ldots J_{h}}$ intersechino al più in un solo punto e in tal caso trasversalmente tale trasformata propria, che se due curve distinte $E_{J_{1} \ldots J_{h}}, E_{J_{1}^{\prime} \ldots J^{\prime}{ }_{a}}$ intersecano ambedue tale trasformata propria siano disgiunte: è ora chiaro che a partire dalla situazione descritta si possa pervenire a quella desiderata.

Osserviamo che se $f: Y \rightarrow S$ è un morfismo di grado 2, $D$ il divisore della ramificazione di $f$ e $\tilde{S}, \tilde{D}$ sono come precisato, il rivestimento doppio $\tilde{f}: \tilde{Y} \rightarrow \widetilde{S}$ che ha $\tilde{D}$ come divisore della ramificazione è ovviamente birazionalmente equivalente a $Y$. Tornando a considerare il morfismo $\varphi: \hat{X} \rightarrow F_{n, r}$ supporremo, con riferimento a quanto sopra, che $S=F_{n, r}$ e che $D=C$ sia un elemento privo di componenti multiple di $\left|-2 K_{F n, r}+2 A\right|=|2 \delta|$ per cui $\tilde{C}=\tilde{D} \in|2 \tilde{\delta}|$ con

$$
\tilde{\delta}=\pi^{*}(\delta)-\sum_{J_{1}} \frac{d_{J_{1}}-\delta_{J_{1}}}{2} E_{J_{1}}-\ldots-\sum_{J_{1} \ldots J_{k}} \frac{d_{J_{1} \ldots J_{k}}-\delta_{J_{1} \ldots J_{k}}}{2} E_{J_{1} \ldots J_{k}}
$$

Sia $\tilde{\varphi}: \tilde{X} \rightarrow \tilde{S}$ il rivestimento doppio di $\tilde{S}$ ramificato lungo $\tilde{C}$. $\tilde{X}$ è non singolare e per ogni $o_{\tilde{s}}$-modulo corrente $\mathfrak{F}$ si ha:

$$
H^{i}\left(\tilde{X}, \tilde{\varphi}^{*} \mathfrak{F}\right) \simeq H^{i}(\widetilde{S}, \mathscr{F}) \oplus H^{i}\left(\tilde{S}, \mathcal{F} \otimes o_{\tilde{S}} o_{S}(-\delta)\right)
$$

In particolare - dato che $K_{\tilde{x}} \equiv \tilde{\varphi}^{*}\left(K_{\tilde{s}}+\tilde{\delta}\right)-$ si ha:

$$
H^{i}\left(\tilde{X} o_{\tilde{x}}\left(n K_{\tilde{X}}\right)\right) \simeq H^{i}\left(\tilde{S} o_{\tilde{S}}\left(n\left(K_{\tilde{s}}+\tilde{\delta}\right)\right)\right) \oplus H^{i}\left(\tilde{S} o_{s}\left(n\left(K_{\tilde{s}}+\tilde{\delta}\right)-\tilde{\delta}\right)\right) .
$$

Vogliamo trovare le condizioni necessarie e sufficienti che deve soddisfare $C$ perchè $\tilde{X}$ sia birazionalmente equivalente a una superficie di Enriques ossia perchè si abbia:

$$
\chi\left(o_{\tilde{x}}\right)=1, \quad h^{0}\left(n\left(K_{\tilde{s}}+\tilde{\delta}\right)\right) \leqslant 1 \quad \forall n \geqslant 0, \quad h^{0}\left(K_{\tilde{s}}+\tilde{\delta}\right)=0, \quad h^{0}\left(2\left(K_{\tilde{s}}+\tilde{\delta}\right)\right)=1
$$


Le condizioni scritte implicano $h^{0}\left(n\left(K_{\tilde{s}}+\tilde{\delta}\right)-\tilde{\delta}\right)=0, \forall n \geqslant 0$ : se fosse $D \in$ $\epsilon\left|n\left(K_{\tilde{s}}+\tilde{\delta}\right)-\tilde{\delta}\right|$ per qualche $n$ si avrebbe $2 D+\tilde{C} \in\left|2 n\left(K_{\tilde{s}}+\tilde{\delta}\right)\right|$ - in particolare $n \geqslant 1-$ e se $\left|2\left(K_{\tilde{s}}+\tilde{\delta}\right)\right|=\{F\}$ dovrebbe essere $n F=2 D+\tilde{C}$, in particolare, dato che $C$ non ha componenti multiple, sarebbe $F^{T} \geqslant \widetilde{C}$ e quindi avremmo $F^{T}-\tilde{C} \in$ $\epsilon\left|2 K_{\tilde{s}}\right|$ il che è impossibile.

i $h^{0}\left(K_{\tilde{T}}+\tilde{\delta}\right)=0$ se e solo se non esiste alcun $A_{0} \in|A|$ tale che

$$
\pi^{*}\left(A_{0}\right)-\sum_{J_{1}}\left(\frac{d_{J_{1}}-\delta_{J_{1}}}{2}-c_{J_{1}}\right) E_{J_{1}} \ldots \sum_{J_{1} \ldots J_{k}}\left(\frac{d_{J_{1} \ldots J_{k}}-\delta_{J_{1} \ldots J_{k}}}{2}-c_{J_{1} \ldots J_{k}}\right) E_{J_{1} \ldots J_{k}} \geqslant 0
$$

ed è $h^{0}\left(2\left(K_{\tilde{s}}+\tilde{\delta}\right)\right)=1$ se e solo se esiste una unica coppia $A_{1}, A_{2}$ di elementi di $|A|$ -necessariamente distinti se $h^{0}\left(K_{\tilde{s}}+\tilde{\delta}\right)=0-$ tale che:

$$
\begin{aligned}
\pi^{*}\left(A_{1}\right)+\pi^{*}\left(A_{2}\right)-2 \sum_{\sigma_{1}}\left(\frac{d_{J_{1}}-\delta_{J_{1}}}{2}-e_{J_{1}}\right) E_{J_{1}} \ldots \\
\ldots-2 \sum_{J_{1} \ldots J_{k}}\left(\frac{d_{J_{1} \ldots J_{k}}-\delta_{J_{1} \ldots J_{k}}}{2}-c_{J_{2} \ldots J_{k}}\right) E_{J_{1} \ldots J_{k}} \geqslant 0 .
\end{aligned}
$$

Supponiamo che sia $h^{0}\left(K_{\dot{s}}+\tilde{\delta}\right)=0$ e $h^{0}\left(2\left(K_{\tilde{s}}+\tilde{\delta}\right)\right)=1$; sia $\Gamma_{i}$ l'insieme dei valori di $J_{1}$ tali che il punto $P_{J_{1}}$ stia su $A_{i}$ (è $\Gamma_{1} \cap \Gamma_{2}=\emptyset$ ). Si può scrivere $\pi^{*}\left(A_{i}\right)=\pi^{-1}\left(A_{i}\right)$
Deve essere

$$
\frac{d_{J_{1}}-\delta_{J_{1}}}{2}-c_{J_{1}}=\frac{n_{J_{1}}-\delta_{J_{1}}}{2}-1 \leqslant 0 \quad \text { se } J_{1} \notin \Gamma_{1} \cup \Gamma_{2}
$$

e

$$
\frac{n_{J_{1}}-\delta_{J_{1}}}{2}-1 \leqslant 1 \quad \text { se } J_{1} \in \Gamma_{1} \cup \Gamma_{2}
$$

in entrambi $i$ casi si ha pertanto $n_{J_{1}} \leqslant 3$.

Dovendo anche essere

$$
\frac{d_{J_{1} J_{2}}-\delta_{J_{1} J_{2}}}{2}-o_{J_{1} J_{2}}=\frac{n_{J_{2}}+n_{J_{1} J_{2}}-\delta_{J_{1} J_{2}}}{2}-2 \leqslant 0 \quad \text { se } J_{1} \notin \Gamma_{1} \cup \Gamma_{2}
$$

si ha anche $n_{J_{1} J_{z}} \leqslant 2$ se $J_{1} \notin \Gamma_{1} \cup \Gamma_{2}$.

Osserviamo che se è $n_{J_{1}} \leqslant 3$ e $n_{J_{1} J_{2}} \leqslant 2$ si ha

$$
\frac{d_{J_{1} \ldots J_{h}}-\delta_{J_{1} \ldots J_{h}}}{2}-C_{J_{1} \ldots J_{h}} \leqslant 0 \quad \forall h \geqslant 2
$$

per cui se fosse $n_{J_{1}} \leqslant 3$ e $n_{J_{1} J_{2}} \leqslant 2, \forall J_{1}, J_{2}$ si avrebbe $h^{0}\left(K_{\tilde{s}}+\tilde{\delta}\right) \neq 0$. Esisteranno perciò delle coppie $J_{1}, J_{2}$ tali che $n_{J_{1}}=n_{J_{1} \sigma_{2}}=3$; se i $P_{J_{1}}$ corrispondenti stessero tutti su $A_{1}$ o tutti su $A_{2}$ l'essere $h^{0}\left(2\left(K_{\tilde{s}}+\tilde{\delta}\right)\right) \neq 0$ equivarrebbe all'essere 
$h^{0}\left(K_{\tilde{s}}+\tilde{\delta}\right) \neq 0$. Possiamo quindi supporre, eventualmente cambiando gli indici che sia $n_{1}=n_{11}=3$ con $P_{1} \in A_{1}$ e $n_{2}=n_{22}=3$ con $P_{2} \in A_{2}$.

Dovendo essere $m_{i i}^{(i)}-\left(n_{i}+n_{i i}-\delta_{i i}-2 c_{i i}\right)=m_{i i}^{(i)}-2 \geqslant 0$ per $i=1,2$ abbiamo che la trasformata propria di $A_{i}$ in $S^{(1)}$ passa per $P_{i i}$ per cui se $A_{i}$ non fosse componente di $O$ la molteplicità di intersezione di $A_{i}$ e $O$ in $P_{i}$ sarebbe almeno 6 mentre è $A_{i} \cdot C=4$.

Dunque $A_{1}$ e $A_{2}$ sono componenti di $C, C-A_{1}-A_{2}$ ha in $P_{i}(i=1,2)$ un punto doppio e la molteplicità di intersezione di $C-A_{1}-A_{2}$ con $A_{i}$ in $P_{i}$ è esattamente 4 ( $C$ non ha componenti multiple e $\left.\left(C-A_{1}-A_{2}\right) A_{i}=4\right)$. In particolare $C-A_{1}-A_{2}$ non ha altri punti in comune con $A_{i}$ all'infuori di $P_{i}$ e si ha $n_{i i J_{3}}<3$ per $i=1,2$ e $\forall_{J_{3}}$.

Osserviamo che le condizioni necessarie ottenute su $C$ perchè sia $h^{0}\left(K_{\tilde{s}}+\tilde{\delta}\right)=0$ $h^{0}\left(2\left(K_{s}+\tilde{\delta}\right)\right)=1$ sono anche chiaramente sufficienti perchè si abbia

$$
h^{0}\left((2 n+1)\left(K_{\tilde{s}}+\tilde{\delta}\right)\right)=0, \quad h^{0}\left(2 n\left(K_{\tilde{s}}+\tilde{\delta}\right)\right)=1 \quad \forall n \geqslant 0 .
$$

Infine per quanto riguarda $\chi\left(o_{\tilde{x}}\right)$ si può scrivere

$$
\begin{aligned}
\chi\left(o_{\tilde{x}}\right)=2 \chi\left(o_{s}\right)+\frac{\tilde{\delta}\left(\tilde{\delta}+K_{\tilde{x}}\right)}{2} & =3+\frac{1}{2} \sum_{J_{1}}\left[\frac{d_{J_{1}}-\delta_{J_{1}}}{2} E_{J_{2}}+\sum_{J_{2}} \frac{d_{J_{1} J_{2}}-\delta_{J_{1} J_{2}}}{2} E_{J_{1} J_{\mathrm{I}}} \ldots\right] \\
\cdot & {\left[\left(\frac{d_{J_{1}}-\delta_{J_{1}}}{2}-C_{J_{1}}\right) E_{J_{1}}+\sum_{J_{2}}\left(\frac{d_{J_{1} J_{2}}-\delta_{J_{1} J_{2}}}{2}-C_{J_{1} J_{2}}\right) E_{J_{1} J_{3}} \ldots\right] . }
\end{aligned}
$$

Se $C$ verifica le condizioni sopra ottenute è facile, benchè tedioso, costruire esplicitamente una $\tilde{S}$ con le proprietà richieste e verificare che se $J_{1} \neq 1,2$ l'addendo relativo a $J_{1}$ nella sommatoria sopra scritta vale 0 mentre se $J_{1}=1$ o 2 esso vale -2 .

Osserviamo da ultimo se $C$ verifica le condizioni ottenute $B$ non può essere una componente di $C$ dato che se lo fosse, essendo $A_{i} B=1, C$ non potrebbe avere in $P_{1}$ e $P_{2}$ le singolarità dette. In particolare deve essere $n \leqslant 2$ dato che se $n>2$ il sistema lineare $|(2 n+4) A+4 B|$ ha $B$ come componente fissa; inoltre se $n>0$, dato che $B C=6-2 n, B$ può passare al più per uno solo dei punti $P_{1}, P_{2}$ e ciò può avvenire solo nel caso che $n=1$.

I risultati di questa sezione sono riassunti nella seguente:

Proposizione 3. - Sia $X$ una superficie di Enriques, $D$ un sistema lineare completo senza componenti fisse e con 2 punti base $F_{1}$ e $P_{2}$ e sia $\varphi_{D}: X \rightarrow F \subseteq \boldsymbol{P}^{d}$ $\left(F=\varphi_{D}(X), d=D^{2} / 2\right)$ la trasformazione razionale ad esso associata. Si ha:

a) $\grave{\mathrm{E}} \operatorname{deg} \varphi_{D}=2$ e $F$ è proiettivamente equivalente a una superficie $F_{n, r}$ con $n+2 r+1=d$ e $n \leqslant 2$.

b) Esiste una curva $E$ tale che il sistema $D+E$ sia senza componenti fisse 
abbia ancora $P_{1}$ e $P_{2}$ come punti base e dia luogo ad una diagramma commutativo

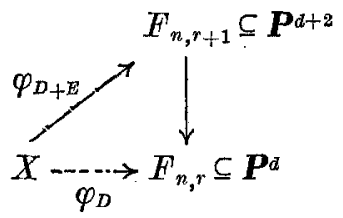

c) Se $r \geqslant 1$ e $C$ è la curva di ramificazione di $\varphi_{D}$ è $C \in|(2 n+6) A+4 B|$, $C$ ha come singolarità al più punti tripli con punti infinitamente vicini del $1^{\circ}$ ordine al più doppi eccezione fatta per 2 punti tripli $Q_{1}$ e $Q_{2}$ con ciascuno un punto infinitamente vicino del $1^{\circ}$ ordine ancora triplo e $\mathrm{i}$ due elementi di $A$ che passano per $Q_{1}$ e $Q_{2}$ sono componenti di $O$. Viceversa se $C$ è un qualunque divisore senza componenti multiple di $|(2 n+6) A+4 B|$ che abbia le proprietà appena specificate, il ricoprimento doppio di $F_{n, r}$ ramificato lungo $C$ è birazionalmente equivalente a una superficie di Enriques.

4. - Su una data superficie $F_{n}$ fissiamo due elementi distinti $A_{1}$ ed $A_{2}$ di $|A|$ e due punti $P_{1}, P_{2}$, tali che $P_{i} \in A_{i}(i=1,2)$.

Sia $F=F_{n}$ e $\pi^{\prime}: F^{\prime} \rightarrow F^{\prime}$ lo scoppiamento di $P_{1}$ e $P_{2}$ : poniamo $E_{i}=\pi^{\prime-1}\left(P_{i}\right)$ $(i=1,2)$. Sia $\pi^{\prime \prime}: \widetilde{F} \rightarrow F^{\prime}$ lo scoppiamento dei punti $P_{1}^{\prime}, P_{2}^{\prime}$ di $E_{1}$ ed $E_{2}$ rispettivamente corrispondenti alle direzioni di $A_{1}$ ed $A_{2}$ e poniamo $E_{i}^{\prime}=\pi^{\prime \prime-1}\left(P_{i}^{\prime}\right)(i=1,2)$. Denoteremo $\pi^{\prime \prime *}\left(E_{i}\right)-E_{i}^{\prime}$ ancora con $E_{i}(i=1,2)$ e porremo $\pi=\pi^{\prime} \circ \pi^{\prime \prime}$. Vogliamo analizzare il sistema lineare completo

$$
\left|((2 n+4) A+4 B)-2 E_{1}-2 E_{2}-4 E_{1}^{\prime}-4 E_{2}^{\prime}\right|=\left|-2 K_{\bar{P}}^{\prime}\right|
$$

di $\widetilde{F}$ nei due casi seguenti: I caso) $B$ non passa nè per $P_{1}$ nè per $P_{2}$ (se $n=0$ ci porremo, come è lecito fare, in tale caso); II caso) $B$ passa per $P_{1}$ ma non per $P_{2}$ e $n=1$. In entrambi i casi, avendo posto $\tilde{A}_{i}=\pi^{*}\left(A_{i}\right)-E_{i}-2 E_{i}^{\prime}(i=1,2)$, e $\tilde{K}=K_{\tilde{F}}$ possiamo scrivere

$$
|-\tilde{K}|=\left|\tilde{A_{1}}+\tilde{A_{2}}+\pi^{*}(n A+B)+\pi_{2} B\right| .
$$

Indicheremo con $C$ una qualunque curva irriducibile non singolare di $\pi^{*}(n A+B)$; $C$ è razionale e si ha $h^{0}(C)=n+2, h^{1}(C)=h^{2}(C)=0$.

Osserviamo che se $\Gamma$ è una curva irriducibile di $\tilde{F}$ tale che $T \cdot \widetilde{K}<0$ si ha senz'altro $h^{1}(\Gamma)=0$ dato che la successione di coomologia associata a

$$
0 \rightarrow o_{\tilde{F}} \rightarrow o_{\tilde{F}}(\Gamma) \rightarrow o_{T}(\Gamma) \rightarrow 0
$$

da $h^{1}(\Gamma)=h^{1}\left(o_{\Gamma}(\Gamma)\right)$ ed essendo per ipotesi $\Gamma^{2}>\Gamma(\Gamma+K)$ è $h^{1}\left(o_{\Gamma}(T)\right)=0$. 
$I$ caso. Sia $\widetilde{B}=\pi^{*} B$. Le suceessioni di coomologia associate alle successioni esatte:

1) $0 \rightarrow o(C) \rightarrow o\left(O+\tilde{A}_{i}\right) \rightarrow o_{A_{i}} A_{i}(-1) \rightarrow 0, \quad i=1,2$

2) $0 \rightarrow o\left(C+\tilde{A}_{i}\right) \rightarrow o\left(C+A_{1}+A_{2}\right) \rightarrow o_{\tilde{A} j}(-1) \rightarrow 0 \quad\{i, j\}=\{1,2\}$

3) $0 \rightarrow o\left(C+\tilde{A_{1}}+\tilde{A_{2}}\right) \rightarrow o\left(O+\tilde{A_{1}}+\tilde{A_{2}}+\tilde{B}\right) \rightarrow o_{\tilde{B}}(2-n) \rightarrow 0$

4) $0 \rightarrow o\left(C+\tilde{A_{i}}\right) \rightarrow o\left(C+\tilde{A}_{i}+\widetilde{B}\right) \rightarrow o_{\tilde{B}}(-n+1) \rightarrow 0$

5) $0 \rightarrow o\left(C+\tilde{A_{i}}+\tilde{B}\right) \rightarrow o\left(C+\tilde{A_{1}}+\tilde{A_{2}}+\tilde{B}\right) \rightarrow o_{\tilde{A}} \rightarrow 0$, $\{i, j\}=\{1,2\}$

mostrano che $h^{1}\left(C+\tilde{A_{i}}\right)=h^{1}\left(C+\tilde{A_{1}}+\tilde{A_{2}}\right)=h^{1}\left(\theta+\tilde{A_{i}}+\widetilde{B}\right)=0$ (successioni 1 , 2 e 4 ), che $h^{0}(-\widetilde{K})=5, h^{1}(-\widetilde{K})=0$ e che nè $A_{1}$ nè $A_{2}$ (successione 5 ) nè $B$ (successione 3) sono parti fisse di $|-\tilde{K}|$. Dunque $|-\tilde{K}|$ non ha parti fisse ed è di conseguenza irriducibile (se fosse composto con un fascio dotrebbe essere $A_{i} \equiv B$ ). Dato che $|C|$ non ha punti base gli eventuali punti base di $|-\tilde{K}|$ dovrebbero essere o su $\tilde{A_{1}}$ o su $\tilde{A_{2}}$ o su $\widetilde{B}$ : poichè si ha $-\tilde{K} \cdot \tilde{A_{i}}=0,-\tilde{K} \cdot \widetilde{B}=n-2$ e poichè la successione 3 mostra che per $n<2|-\widetilde{K}|$ sega su $\widetilde{B}$ una serie completa di grado $2-n$ necessariamente priva di punti fissi possiamo concludere che $|-\tilde{K}|$ non ha punti base.

Di conseguenza $|-2 \tilde{K}|$ non ha parti fisse, è irriducibile e non ha punti base; essendo $(-2 \tilde{K}) \tilde{K}=-8<0$ si ha $h^{1}(-2 \widetilde{K})=0$ e $h^{0}(-2 \tilde{K})=\chi(-2 K)=13$.

$I I$ caso. Sia $\widetilde{B}=\pi^{*} B-E_{1}-Z_{1}^{\prime}$. Le successioni di coomologia associate a:

$$
\begin{aligned}
& 0 \rightarrow o(O) \rightarrow o\left(O+E_{\mathfrak{1}}^{\prime}\right) \rightarrow o_{E_{1}^{\prime}}^{\prime}(-1) \rightarrow 0 \\
& 0 \rightarrow o\left(O+A_{1}\right) \rightarrow o\left(O+A_{1}+E_{1}^{\prime}\right) \rightarrow o_{E_{1}^{\prime}} \rightarrow 0
\end{aligned}
$$

mostrano che $\left|C+A_{1}+E_{1}^{\prime}\right|$ non ha parti fisse: se ne può dedurre che è irriducibile e senza punti fissi. Sia $C^{\prime}$ un elemento irriducibile non singolare di $\left|O+A_{1}+E_{1}^{\prime}\right|$; $O^{\prime}$ è razionale dato che $O^{\prime 2}=n+1$ e $O^{\prime} \tilde{K}=-3-n$. Se si ripetono le considerazioni fatte nel caso I mettendo $C^{\prime}$ al posto di $C$ ed $E_{1}$ al posto di $\tilde{A_{1}}$ si trova che $|-2 \tilde{K}|$ è privo di parti fisse, irriducibile e senza punti base e che si ha ancora $h^{0}(-2 \tilde{K})=13, h^{1}(-2 \tilde{K})=0$.

Abbiamo così esaurito l'analisi del sistema $-2 \tilde{K}$ nei due casi che ci eravamo prefissi.

Supponiamo ora di trovarci in uno dei due casi descritti e sia $T$ un elemento irriducibile non singolare di $|-2 K|$. Dato che $\Gamma E_{i}=\Gamma \cdot \tilde{A_{i}}=E_{1} \cdot E_{2}=\tilde{A}_{1} \cdot \tilde{A}_{2}=E_{i} \tilde{A}_{J}=0$ la curva $\Delta=\Gamma+\tilde{A_{1}}+\tilde{A_{2}}+E_{1}+E_{2}$ è non singolare e si ha

$$
\Delta \in\left|-2\left(\tilde{K}+E_{1}^{\prime}+E_{2}^{\prime}-\pi^{*}(A)\right)\right|
$$

Sia $\varrho: \tilde{X} \rightarrow \tilde{F}$ il rivestimento doppio di $F$ ramificato lungo $\Delta$. Se $\varrho^{*}\left(\tilde{A_{i}}\right)=2 O_{i}$ $(i=1,2), \varrho^{*}\left(E_{i}\right)=2 C_{i \uparrow^{2}}(i=1,2)$, le $C_{J}$ sono 4 curve eccezionali del $1^{\circ}$ genere. 
Sia $\varepsilon: \tilde{X} \rightarrow X$ la superficie ottenuta contraendole. $X$ è una superficie di Enriques; in effetti si ha:

$$
2 K_{\tilde{X}} \equiv \varrho^{*}\left(\tilde{A_{1}}+\tilde{A}_{2}+E_{1}+E_{2}\right)=2 \sum_{i=1}^{4} C_{i}=\varepsilon^{*}\left(2 K_{X}\right)+2 \sum_{i=1}^{4} C_{i}
$$

cosicchè $2 K_{X} \equiv 0$, e inoltre è

$$
\chi\left(o_{x}\right)=\chi\left(o_{\tilde{x}}\right)=2 \chi\left(o_{\tilde{p}}\right)+\frac{\tilde{\delta}(\tilde{\delta}+\tilde{K})}{2}=2-1=1 .
$$

Fissiamo un $r \geqslant 0$ con $r>0$ se $n=0$ e poniamo $\widetilde{D} \equiv \varrho^{*} \pi^{*}((n+r) A+B)$; ̀̀ $\tilde{D}^{2}=2(n+2 r), \tilde{D} C_{1}=\tilde{D} C_{2}=1, \tilde{D} C_{3}=\tilde{D} C_{4}=0$ per cui se $\varepsilon_{*} \tilde{D}=D$ è $D^{2}=$ $=2(n+2 r)+2$. Il morfismo $\pi \circ \varrho$ induce una trasformazione razionale $T: X \rightarrow F_{n}$; essendo $h^{0}(D)=n+2 r+2$ è $\varphi_{n, r} \circ T=\varphi_{D}$.

Osserviamo che se $\Gamma$ è un qualsiasi elemento irriducibile di $|-2 \tilde{K}|$ il ricoprimento doppio di $\tilde{F}$ ramificato lungo $\Delta=\Gamma+\tilde{A_{1}}+\tilde{A_{2}}+E_{1}+E_{2}$ è birazionalmente equivalente a una superficie di Enriques dato che $\Gamma$, essendo $P_{a}(T)=5$, può avere come singolarità al più un punto triplo con un punto doppio infinitamente vicino del $1^{\circ}$ ordine.

Richiamiamo ora la descrizione del gruppo $G_{n}$ degli automorfismi di una superficie $F_{n}$.

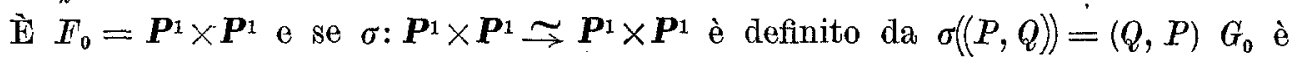
generato da $\sigma$ e dal suo sottogruppo normale $P G L(1) \times P G L(1), P G L(1)$ essendo il gruppo degli automorfismi di $\boldsymbol{P}^{\mathbf{1}}$. Se $n \geqslant 1$, riferendoci alla realizzazione di $F_{n}$ come fibrato su $\boldsymbol{P}^{1}$ descritta nella sezione 2 si ha che ogni $g \in G_{2}$ induce un automorfismo della base: la mappa $G_{n} \rightarrow P G L(1)$ così ottenuta è suriettiva e se $N_{n}$ ne è il nucleo $H_{n}$ può essere identificato con il gruppo quoziente del gruppo moltiplicativo di matrici

$$
\left\{\left(\begin{array}{ll}
a^{-1} & 0 \\
C(t) & a
\end{array}\right) \mid a \in C^{*}, C(t) \in C[t] \quad \text { e } \quad \operatorname{deg} C(t) \leqslant n\right\}
$$

modulo il suo sottogruppo

$$
\left\{\left(\begin{array}{rr} 
\pm 1 & 0 \\
0 & \pm 1
\end{array}\right)\right\}
$$

L'azione di $H_{n}$ sull'aperto $\boldsymbol{P}^{1}-\{\infty\} \times \boldsymbol{P}^{1}$ di $F_{n}$ può essere descritta esplicitamente cosi:

$$
\left(t ; X_{0}: X_{1}\right) \mapsto\left(t, a X_{0}: C(t) X_{0}+a^{-1} X_{1}\right)
$$

In particolare si ha che prese comunque $n+1$ fibre distinte $A_{0} \ldots A_{n}$ e prese comunque $n+2$ coppie di punti $\left(P_{-1}, P_{-1}^{\prime}\right),\left(P_{0}, P_{0}^{\prime}\right), \ldots,\left(P_{n}, P_{n}^{\prime}\right)$, nessuno dei quali 
stia su $B$ e tali che $P_{i}, P_{i}^{\prime} \in A_{i}, i=0, \ldots, n . P_{-1}, P_{-1}^{\prime} \in A_{0}, P_{-1} \neq P_{0}, P_{1}^{\prime} \neq P_{0}^{\prime}$ esiste uno ed un solo elemento $h \in H_{n}$ tale che $h\left(P_{J}\right)=P_{J}^{\prime}, \forall J \geqslant-1$.

Consideriamo gli insiemi $I_{0}=\left\{\{P, Q\} \mid P, Q \in F_{0}, P \neq Q\right\}, I_{1}=\left\{\{P, Q\} \mid P, Q \in F_{1}\right.$, $P$ e $Q$ non stanno su uno stesso elemento di $A$ nè stanno entrambi su $B\}$, $I_{2}=\left\{\{P, Q\} \mid P, Q \in F_{2}, P\right.$ e $Q$ non stanno nè su uno stesso elemento di $A$ nè su uno stesso elemento di $B\}$; facciamo agire $G_{n}$. su $I_{n}$ ponendo $g(\{P, Q\})=\{g(P), g(Q)\}$ se $g \in G_{n}$ e $\{P, Q\} \in I_{n}(n=0,1,2)$. Da quanto richiamato risulta immediatamente che: $I_{0}$ si ripartisce per l'azione di $G_{0}$ in due orbite, una, che denoteremo $O_{1}^{(0)}$ costituita dagli elementi $\{P, Q\}$ tali che $P$ e $Q$ non stanno nè su uno stesso elemento di $A$ nè su unó stesso elemento di $B$, l'altra, $O_{2}^{(0)}$, costituita dai restanti elementi di $I_{0}$; $I_{1}$ si ripartisce anch'esso per l'azione di $G_{1}$ in due orbite, una $O_{1}^{(1)}$ costituita dagli elementi $\{P, Q\}$ tali che nè $P$ nè $Q$ stanno su $B$, l'altra $O_{2}^{(1)}$ costituita dagli altri elementi di $I_{1}$.

$G_{2}$ agisce transitivamente su $I_{2}$.

Se $S_{n}(\{P, Q\})$ indica lo stabilizzatore di $\{P, Q\} I_{n}$ per l'azione di $G_{n}(n=0,1,2)$ si ha dunque:

$$
\begin{array}{llll}
\operatorname{dim} S_{0}(\{P, Q\})=2 & \text { se }\{P, Q\} \in O_{1}^{(0)} & \operatorname{dim} S_{0}(\{P, Q\})=3 & \text { se }\{P, Q\} \in O_{2}^{(0)} \\
\operatorname{dim} S_{1}(\{P, Q\})=2 & \text { se }\{P, Q\} \in O_{1}^{(1)} & \operatorname{dim} S_{0}(\{P, Q\})=3 & \text { se }\{P, Q\} \in O_{2}^{(1)} \\
\operatorname{dim} S_{2}(\{P, Q\})=3 & \text { se }\{P, Q\} \in I_{2} . & &
\end{array}
$$

In definitiva $i$ calcoli fatti nel corso di questa sezione permettono di asserire la seguente

Proposizione 4. - La dimensione dello "spazio dei moduli" delle superficie di Enriques $X$ che hanno un sistema lineare $D$ senza parti fisse tale che la trasformazione razionale ad esso associata, $D$, abbia per immagine $F_{n}$ è 10 se $n=0,1$ ed è 9 se $n=2$.

\section{BIBLIOGRAFIA}

A. Beadville, Surfaces algebriques complexes, Astérisque, 54 (1978).

I. Chafarevitch ed altri, Algebraic surfaces, Proc. of the Steklov Institute, 75 (1965).

F. ExRrques, Sopra le superficie algebriche di bigenere uno, Memorie della Società Italiana delle Scienze, serie $3^{\mathrm{a}}, 14(1906)$.

F. ENRIQUES, Sopra le superficie algebriche di bigenere uno, Memorie dellà Società Italiana delle Scienze, serie $3^{\mathrm{a}}$, 14 (1906).

M. Nagata, On rational surfaces. - I: Irreducible curves of arithmetic genus 0 or 1 , Mem. Coll. Sci. Kyoto Ser. A Math., 32 (1960).

M. REID, Hyperelliptic linear systems on a K3 surface, J. London Math. Soc., (2), 13 (1976). 